\title{
Objetivos del sistema de salud en México: importancia para la población y sus determinantes
}

\author{
José Antonio Tapia-Cruz, M en C.11
}

\section{Tapia-Cruz JA. \\ Objetivos del sistema de salud en México: importancia para la población y sus determinantes. Salud Publica Mex 2006;48:113-126.}

\section{Resumen}

Objetivo. Documentar la importancia que tienen para la población los cinco objetivos intrínsecos del sistema de salud (SS) actual en México y describir algunas de las determinantes de su valoración; la finalidad es aportar elementos para definir prioridades y promover el cumplimiento de dichos objetivos. Material y métodos. Este es un estudio transversal sobre la importancia para la población mexicana de los cinco objetivos del SS, considerados intrínsecos 0 finales en la Encuesta $\mathrm{N}$ acional de Evaluación del Desempeño 2002. La variable dependiente fue la posición que la población encuestada asignó a cada uno de los objetivos del SS con base en la pregunta: "icuál objetivo del SS le parece el más importante?". Las variables independientes (nominales y categóricas) fueron: entidad federativa, tipo de localidad, sexo, edad, escolaridad, empleo actual, calificación del estado de salud actual, última o casión en que necesitó asistencia médica y no la recibió, grado de satisfacción con el funcionamiento de la atención médica en el país y satisfacción con su salud. Se realizó un análisis univariado que se resumió mediante porcentajes. Se utilizó la prueba de la ji cuadrada para validar o rechazar la hipótesis según la cual la posición asignada por la población a los objetivos del SS es la misma, sin importar cuáles fueran las variables utilizadas en la comparación, y se desarrolló un modelo de regresión logística multinomial (politómica) para correlacionar el efecto de las variables independientes empleadas en el estudio, en relación con la importancia asignada por la población a los objetivos del SS. La categoría de referencia del modelo fue el objetivo de mejorar la salud de la población. El análisis se realizó con el paquete estadístico STATA versión 7.0. Resultados El 31.8\% de la población

\author{
Tapia-Cruz JA. \\ Mexico health care system's objectives: \\ importance to its population and determining factors. \\ Salud Publica Mex 2006;48:113-126.
}

\begin{abstract}
A bstract
Objective. To document the importance to Mexico 's population of the five intrinsic objectives of Mexico's present health care system (SS) and describe some of the determinants of its evaluation; the purpose is to contribute elements for defining priorities and to support the fulfillment of said objectives. Material and Methods. This is a crosssectional study to the importance for Mexican population of the five objectives of SS, considered intrinsic or final in the $\mathrm{N}$ ational Evaluation and Performance Survey. The dependent variable was the ranking given by the population surveyed to each one of the objectives, based on the question:W hich SS objective do you believe is most important? The independent variables (nominal and categorical) were: state of residence, type of locality, gender, age, education, present employment, quality of present state of health, last occurrence when medical assistance was needed but not received, grade of satisfaction with the operation of the country's medical care system and satisfaction with one's own health. A univaried analysis was conducted and summarized using percentages. The chi square test was used to validate or reject the hypothesis according to whether the rank assigned by the population to the SS objectives is the same, regardless of which variables were used in the comparison.A multino mial (politomic) logistical regression model was also developed to correlate the effect of the independent variables employed in the study with the importance assigned by the population to the SS objectives. The reference category of the model was the objective of improving the health of the population. The analysis was conducted using the statistical package STATA version 7.0. Results. From the population studied, $31.8 \%$ reported that the most
\end{abstract}

(1) Subdirección de Evaluación de Servicios No Personales de Salud; Dirección General de Evaluación del Desempeño. Secretaría de Salud. México.

Fecha de recibido: 2 de julio de 2004 • Fecha de aprobado: 19 de diciembre de 2005

Solicitud de sobretiros: José Antonio Tapia Cruz. Dirección General de Evaluación y Desempeño. Secretaría de Salud. Reforma N 0.450, piso 12, colonia Juárez, 06600 México, D.F., México.

Correo electrónico: jatapia@ salud.gob.mx 
de estudio considera que mejorar el trato dentro del sistema de salud es el objetivo más importante . En el área rural, $31.46 \%$ de la población piensa que el objetivo más importante es mejorar la salud, un resultado relacionado con la auto percepción del estado de salud actual y la falta de atención médica. La probabilidad de preferir el objetivo de mejorar el trato en lugar de mejorar la salud entre la población con educación media y superior es 10 y $16 \%$ más alta, respectivamente, en comparación con esa misma probabilidad para la población sin educación. Entre la población que trabaja de manera independiente, $31.2 \%$ concede más importancia al objetivo de mejorar la salud, porcentaje mayor al que notifica la población considerada como empresaria 0 constituida por empleados públicos y privados. Conclusiones. En términos generales, la población analizada en este estudio consideró más importante mejorar el trato dentro del sistema de salud, lo cual difiere del planteamiento establecido en el PRO N ASA 2001-2006. Se detectó que la importancia asignada a los objetivos puede variar según sean el grupo que se analice, sus características y la influencia que ejercen diversos factores externos sobre el individuo, con lo cual se rechaza la hipótesis nula. La variación entre las valoraciones de la población se presentó sobre todo entre el objetivo de mejorar el trato dentro del SS y el de mejorar la salud de la población. Los resultados obtenidos guían las medidas de comunicación y for talecimiento del SS para adecuarse a las expectativas de la población y favorecer el cumplimiento de los objetivos establecidos.

Palabras clave: evaluación de programas; objetivos organizacionales; satisfacción de los consumidores; administración y planificación en salud; México important objective is to improve treatment within the health care system. In rural areas, $31.46 \%$ of the po pulation thinks the most important objective is to improve health, a result related with the self-perception of one s present state of health and lack of medical care.The probability of preferring the objective of improving treatment over improving health, between the populations with average education and higher levels of education is, respectively, $10 \%$ and $16 \%$ greater than the population with no education. A mong the population that works independently, $31.2 \%$ consider improving health the most important objective, a greater percentage than that reported by the population of business owners and the one consisting of public and private employees. Conclusions. In general terms, the population analyzed by this study considered the improvement of treatment within the health care system to be most important, which differs from that suggested by the PRO N ASA 2001-2006. It was indicated that the importance assigned to the objectives can vary according to the group analyzed, its character istics and the influence of diverse external factors on the individual, as a result of which the null hypothsesis is rejected. Variations among the populations' evaluations occurred, in particular, between the objective of improving treatment within the health care system and that of the improvement of the population's health.The obtained results guide the steps for communication and the strengthening of the health care system in order to adjust itself to the expectations of the population and support the fulfillment of the established objectives.

Key words: program evaluation; organizational objectives; consumer satisfaction; health administration and planning; Mexico
L a conformación de los sistemas de salud (SS) y su operación, como cualquier otro sistema, se sustentan en principios y valores fundamentales que comparten los individuos en sociedad. En esencia, su organización y administración buscan mantener ese núcleo de valores y cumplir los objetivos que cohesionan a la sociedad y permiten satisfacer sus necesidades y solucionar los conflictos, dada la diversidad de grupos, individuos e intereses específicos. ${ }^{1}$

El desarrollo de políticas públicas es el mecanismo de los gobiernos para transformar las exigencias de la población y solucionar sus demandas. En México, el Plan Nacional de Desarrollo 2001-2006 señala que las políticas públicas son el conjunto de concepciones, criterios, principios, medidas y líneas fundamentales de acción, con los cuales la comunidad organizada como Estado hace frente a desafíos y problemas que se consideran de naturaleza pública. ${ }^{2}$ Por ello, la definición explícita de los objetivos que buscan alcanzar los sistemas que integran al Estado -actividad que forma parte de la función rectora de éste- permite orientar el sentido y contenido de las políticas públicas, bajo las cuales se generan planes y programas (como el de salud) que suponen la generación y asignación de recursos, la operación de servicios, la aplicación de leyes y normas, y la rendición de cuentas.

En consecuencia, la creación de los SS, tal y como se conocen ahora, es la respuesta social organizada ante el fenómeno de la salud. Se trata de sistemas complejos, por las múltiples interacciones en su interior y con otros sistemas de la sociedad, ${ }^{3}$ y dinámicos, al evolucionar con los avances en el terreno de la salud, adecuándose al cambio de necesidades y expectativas de la población. Hoy es innegable la influencia de los SS sobre las condiciones de salud de la población y el desarrollo de las sociedades. En cuanto a la atención, por ejemplo, las personas se interrelacionan en la actualidad con los SS en busca de ayuda para un número de problemas y necesidades mucho mayor del concebible hace más de un siglo, cuando la atención de la salud 
estaba confinada en mayor medida al ámbito familiar. ${ }^{4}$ Sin embargo, la salud es, además, un bien fundamental del desarrollo y bienestar social, y un sector creciente de la economía. ${ }^{5}$

Durante su evolución, los SS han adoptado diversas formas y modos de operar; empero, el principio rector que los origina y guía es el de proteger, mejorar y restablecer la salud de los individuos; sin él los SS no tendrían razón de ser. Dicho objetivo consiste, primero, en lograr el mayor nivel posible de salud entre la población y, segundo, en reducir las diferencias (desigualdades) de salud entre los grupos poblacionales, es decir, lograr la equidad de las condiciones de salud. Más aún, de acuerdo con la Organización Mundial de la Salud (OMS), existen otros objetivos fundamentales que deben considerar los SS, como cualquier otro sistema social : a) responder a las expectativas legítimas de la población y b) lograr la equidad en la contribución financiera que los sistemas requieren para funcionar. ${ }^{6}$

La capacidad de respuesta del sistema se refiere a la forma en que las instituciones y los actores del proceso se interrelacionan con la población para atender sus demandas y expectativas. Esto implica, por un lado, otorgar un trato adecuado que considere el respeto a las personas (trato digno, confidencialidad, comunicación y autonomía) y servicios orientados al usuario (atención pronta, acceso a redes sociales de apoyo, comodidades dentro de las unidades de atención y selección del proveedor) y, por el otro, disminuir las diferencias entre la población respecto de la atención. Por su parte, la equidad en la contribución financiera busca garantizar esquemas de financiamiento justos para que los hogares más pobres paguen menos que los más ricos, además de evitar que las familias se empobrezcan o paguen sumas excesivas por recibir atención de salud. ${ }^{6}$

La definición y establecimiento de estos objetivos por parte de la OMS suscitó diversas consultas regionales en todo el mundo y un amplio debate. En todos los casos resultó unánime que mejorar la salud era el objetivo final e intrínseco de los SS. No obstante, la pertinencia del trato adecuado y la protección financiera como objetivos finales de los SS no obtuvieron el mismo consenso, ya que ambos pueden considerarse objetivos finales de los sistemas, pero también atributos deseables del sistema o metas intermedias. Tales consideraciones dejaron en claro la necesidad de generar mayor evidencia empírica, a partir del análisis de las preferencias de la población en contextos determinados. Con esa finalidad se desarrollaron encuestas poblacionales acerca de la importancia de los objetivos definidos por el sistema de salud en cada país.?

\section{Los objetivos de salud en México}

En México, como parte del Programa Nacional de Salud 2001-2006 (PRONASA 2001-2006), ${ }^{8}$ se plantearon los siguientes objetivos:

- Mejorar las condiciones de salud de los mexicanos.

- Abatir las desigualdades en salud.

- Garantizar un trato adecuado en los servicios públicos y privados de salud.

- Asegurar la justicia en el financiamiento en materia de salud.

- Fortalecer el Sistema Nacional de Salud.

Estos, que guardan concordancia con los establecidos por la OMS -con excepción del último- y con los objetivos del Plan Nacional de Desarrollo 2001-2006 relacionados con el desarrollo social y humano, buscan responder a los retos de equidad, calidad y protección financiera que enfrenta hoy en día el SS en el país. Para lograrlo se formularon 10 estrategias -cinco sustantivas por su relación directa con la consecución de los objetivos básicos del sistema y cinco instrumentales para fortalecerlo- vinculadas con las funciones básicas del sistema: prestación de servicios, generación de recursos, financiamiento y rectoría.

En el PRONASA 2001-2006 se planteó además la necesidad de crear mecanismos para evaluar el cumplimiento de estos objetivos, valorar el funcionamiento general del SS y fortalecer la rendición de cuentas dentro del sector, puesto que la evaluación es una actividad básica de la función rectora de la Secretaría de Salud (SSA). ${ }^{9}$ Por ello, la SSA aplica procesos de evaluación del desempeño con objeto de medir el grado en el que se alcanzan los objetivos intrínsecos del sistema en sus ámbitos nacional y estatales. De igual modo, valora los logros obtenidos en relación con lo que puede esperarse de los SS, a partir de los recursos de los que dispone, y aporta elementos para suministrar servicios, tomar decisiones y ajustar las políticas.

La aplicación de la metodología de evaluación del desempeño del SS en México inició con el análisis del actual sistema de información en salud. Se reconoció que éste no proporciona datos sobre capacidad de respuesta a las expectativas no médicas de las personas (trato adecuado) y de justicia en el financiamiento (protección financiera). Por ello se planteó la necesidad de desarrollar en el 2002 la Encuesta Nacional de Evaluación del Desempeño (ENED 2002). ${ }^{10}$

Cabe señalar que la ENED 2002 forma parte, además, de un convenio de colaboración con la OMS para desarrollar y probar instrumentos de evaluación del 
desempeño de los sistemas de salud, por lo cual la encuesta permite comparar sus resultados con los de otros países. Sin embargo, por esa misma razón no se analizó el objetivo de fortalecer el sistema nacional de salud definido en el PRONASA 2001-2006 y sólo se obtuvo información sobre los llamados objetivos intrínsecos o finales del sistema, que corresponden a los cuatro primeros objetivos del sistema de salud mexicano.

A partir de los resultados de la encuesta, este trabajo se justifica porque aporta información empírica sobre los objetivos finales del SS en el país. El objetivo de este trabajo es documentar la relevancia que tienen para la población los cinco objetivos intrínsecos del actual SS y describir algunas de las determinantes que intervienen en su valoración; la finalidad es suministrar elementos para la definición de prioridades y el cumplimiento de dichos objetivos.

\section{Material y métodos}

Se desarrolló un estudio transversal en relación con la importancia que tienen para la población mexicana los cinco objetivos del SS, considerados como intrínsecos o finales en la ENED 2002: a) mejorar la salud de la población, que implica aumentar el nivel global de salud de la población; b) minimizar las diferencias, en cuanto a las condiciones de salud, que separan a los grupos poblacionales; c) mejorar la capacidad de respuesta del sistema de salud, que en este estudio equivale a mejorar el trato; d) reducir al mínimo las desigualdades en la capacidad de respuesta, para lo que deben abatirse las diferencias en el trato concedido a los grupos poblacionales, y e) lograr la equidad en la contribución financiera, lo cual supone que los hogares aporten, para financiar al SS, una cantidad de recursos proporcional a su ingreso, incluida la exención del pago.

La fuente de información utilizada fue la ENED 2002. De ella se recogieron datos sobre la percepción general de las condiciones de salud de los mexicanos, el trato que recibe la población en las instituciones de salud, el nivel de protección financiera del sistema y la importancia que confiere la población a los objetivos del SS en México. Es importante mencionar que en la encuesta se empleó el cuestionario que la OMS utilizó como piloto en varios países durante el año 2001 y que se adecuó a las características de México. En el artículo "Metodología de la encuesta nacional de evaluación del desempeño" se describen de forma exhaustiva las características de la ENED 2002 y el proceso de obtención de la información; ${ }^{11}$ empero, es necesario referir que la ENED 2002 tiene representatividad nacional, estatal, rural y urbana, y que se aplicó a 38746 hogares (cerca de 1250 por estado); esta cifra, mediante el ponderador definido durante su planeación, permite extrapolar los resultados a 75 millones 742 mil 297 personas en el plano nacional.

La variable dependiente analizada fue la posición que la población encuestada asignó a cada uno de los cinco objetivos del SS considerados como intrínsecos o finales, con base en la pregunta: ¿cuál objetivo del SS le parece el más importante? Las variables independientes, algunas nominales y otras categóricas, fueron: entidad federativa; tipo de localidad: rural $(<2500$ habitantes) y urbana (2 500 o más habitantes); sexo (masculino o femenino); edad, con los grupos de 17 a 24 años, 25 a 59 y 60 o más; escolaridad: a) ninguna, b) educación básica (primaria y secundaria), c) educación media (preparatoria, bachillerato y carrera técnica) y d) educación superior (licenciatura, maestría y doctorado); empleo actual, clasificado en "trabaja y no trabaja", y una distinción por actividad laboral actual cuyas categorías fueron empleado público, empleado particular, trabajador por su cuenta, empresario, trabajador no remunerado (voluntario) y sin trabajo actual.

Otras variables incluidas fueron autopercepción del estado de salud actual, clasificado como bueno, regular y malo; última ocasión en que se necesitó asistencia médica y no se recibió (en los últimos 30 días o más de un mes pero menos de un año); satisfacción de la persona con el funcionamiento de la atención médica en el país (satisfecho, regular o insatisfecho); y la valoración de la propia salud (satisfecho, regular e insatisfecho).

Se realizó un análisis univariado que se resumió mediante porcentajes. Se usó la prueba de la ji cuadrada para validar o rechazar la hipótesis según la cual la posición asignada por la población a los objetivos del SS es la misma, sin importar cuáles fueran las variables utilizadas en la comparación, y por último, se desarrolló un modelo de regresión logística multinomial (politómica) para correlacionar el efecto de las variables independientes empleadas en el estudio, con la importancia asignada por la población a los objetivos del SS. La categoría de referencia del modelo fue el objetivo de mejorar la salud de la población, dado que éste se considera el objetivo definitorio del SS. El análisis se realizó con el paquete estadístico STATA versión 7.0.

\section{Resultados}

\section{Descripción de variables}

La población de estudio, de acuerdo con los resultados ponderados de la ENED 2002, asciende a 75 millones 742 mil 297 habitantes en el país, de los cuales 77\% vive en áreas urbanas y $23 \%$ en zonas rurales. Su distribu- 
ción por sexo señala que $58 \%$ correspondió a mujeres y el resto a hombres. Por grupo de edad se observó que $67.1 \%$ se incluyó en el grupo de 25 a 59 años de edad, $18.9 \%$ en el de 17 a 24 años y $14 \%$ en el de 60 años o más, una distribución demográfica similar a la nacional.*

Del total de población de estudio, $13 \%$ refirió no contar con educación formal, $63.3 \%$ tenía educación básica, $15.6 \%$ había cursado educación media y $8.2 \%$ educación superior. Por otra parte, durante el periodo de aplicación de la encuesta, 39 millones 5 mil 986 personas expresaron no tener un trabajo formal (incluidos los que no trabajaban y los que realizaban alguna actividad laboral sin percibir ningún salario), lo que representa $51.5 \%$ del total de la población de estudio. La población que tenía empleo formal sumaba $36 \mathrm{mi}$ llones 736 mil 311 personas (48.5\%), de las cuales 18\% trabajaba en el sector público, $32.3 \%$ en la iniciativa privada, $49.2 \%$ por su cuenta y $0.5 \%$ era empresario.

En cuanto a las variables relacionadas con la salud y los servicios de atención, en la ENED 2002 se registró que 50 millones 142 mil 872 habitantes consideraban su salud actual como buena, 21 millones 512 mil 367 como regular y 4 millones 87 mil 058 como mala. Por su parte, del total de la población de estudio, 1.6\% señaló que no había requerido asistencia médica. De los que sí la necesitaron (74 millones 519 mil 103), 97.5\% la recibió, $2.4 \%$ no y $0.1 \%$ no supo. La población que requirió atención médica en el último mes y no la obtuvo ascendió a 819 mil 37 habitantes, mientras que 470 mil 914 personas, pese a necesitar atención en un periodo mayor de 30 días pero menor de un año, no la recibieron.

La satisfacción de la población con el funcionamiento de la atención médica en el país, según la ENED 2002 , revela que $44.6 \%$ se encuentra satisfecho, $35.2 \%$ sólo de cierta forma y $20.2 \%$ no lo está. Por último, $60.8 \%$ de la población está satisfecho con su salud, $21.1 \%$ en algún grado y $18.1 \%$ no lo está.

\section{Importancia de los objetivos del SS para la población de México y sus determinantes}

Durante la aplicación de la ENED 2002, se le solicitó a la población seleccionada que precisara cuál de los cinco objetivos establecidos en ella le parecía el más importante del sistema de salud; para ello, durante la aplicación de la encuesta, se le explicó a la población en qué consistía cada uno de los objetivos analizados del SS.

\footnotetext{
* Consejo Nacional de Población. Proyecciones de población 20002050. México: Consejo Nacional de Población, 2002. Documento no publicado.
}

Con las respuestas recogidas se obtuvieron los porcentajes de población de acuerdo con el objetivo más importante y se observó el siguiente orden para la población en general : a) mejorar el trato dentro del SS (31.8\% de la población encuestada), b) mejorar la salud de la población $(29.5 \%)$, c) minimizar las diferencias entre las personas respecto de la salud (17.4\%), d) reducir las desigualdades en la capacidad de respuesta del sistema (13\%), y e) lograr la equidad en la contribución financiera dentro del SS (8.3\%). Este orden se aparta del plasmado en el PRONASA 2001-2006, en el que las posiciones son las siguientes: a) mejorar las condiciones de salud de los mexicanos, b) abatir las desigualdades en salud, c) garantizar un trato adecuado en los servicios públicos y privados de salud y d) asegurar la justicia en el financiamiento en materia de salud.

Debido a este cambio de posiciones, se trató de identificar los posibles determinantes que influían en la población de estudio para asignar dicho orden; tras analizar diversas variables contenidas en la ENED 2002, se obtuvieron los siguientes resultados.

$\mathrm{Al}$ revisar el orden de los objetivos analizados en la ENED 2002 por entidad federativa, se advirtió que en 16 estados se mantuvo el orden informado por la población nacional, mientras que en Baja California, Chiapas, Durango, Guanajuato, Guerrero, Hidalgo, Nayarit, Oaxaca, Querétaro, San Luis Potosí, Tamaulipas y Tlaxcala, el objetivo de mejorar la salud de la población se consideró el más importante del sistema, antes que mejorar el trato dentro del SS. Como se observa en la figura 1, que esas entidades prefieran el objetivo de mejorar la salud se relaciona con la percepción del estado de salud actual que tiene la población, ya que con la excepción de Guerrero y Oaxaca, los porcentajes de población que consideran su salud como buena son menores que los de otras entidades; por el contrario, los porcentajes de población que valoran su estado de salud actual como regular y malo son mayores. De acuerdo con la prueba de la ji cuadrada $(\operatorname{Pr}=0.000)$, se establece que la importancia concedida por la población a los objetivos del SS depende de la entidad de residencia y la percepción del estado de la salud actual, con lo cual se rechaza la hipótesis según la cual la valoración de los objetivos es la misma al margen de la variable incluida en el análisis.

Por otra parte, la importancia otorgada a los objetivos del SS entre la población de las áreas urbana y rural muestra que, para la población rural, el objetivo más importante es mejorar la salud de la población y, en segundo, mejorar el trato. Entre la población urbana la posición de los objetivos coincide con la nacional. La preferencia por mejorar la salud en las áreas 


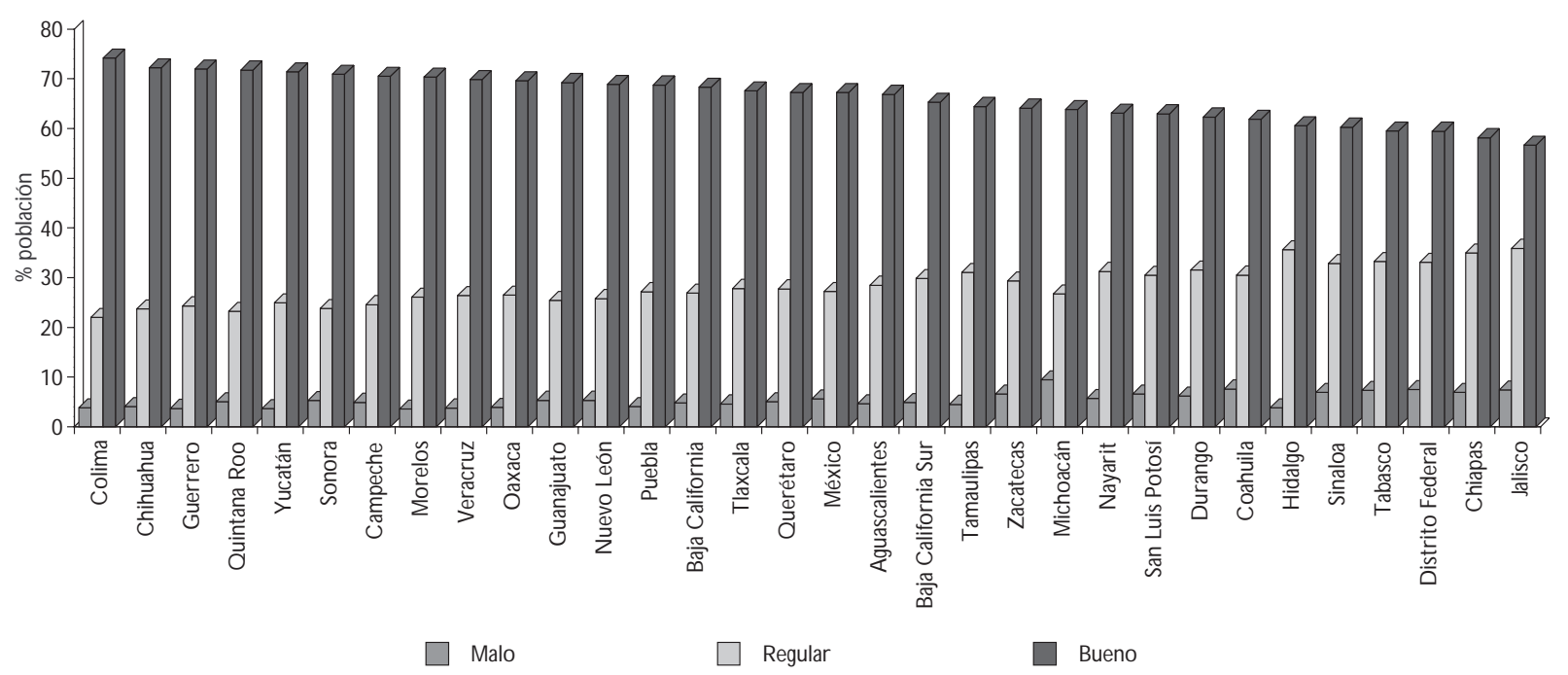

Fuente: Encuesta N acional de Evaluación del Desempeño, 2002. Dirección General de Información y Evaluación del Desempeño; Secretaría de Salud

Figura 1. Autopercepción del estado de salud actual por entidad federativa. México, 2002

rurales concuerda con los informes de otros indicadores que reflejan que los niveles de salud de la población rural son bajos en relación con la media nacional y con los de las áreas urbanas. ${ }^{10}$ Esto, según la ENED

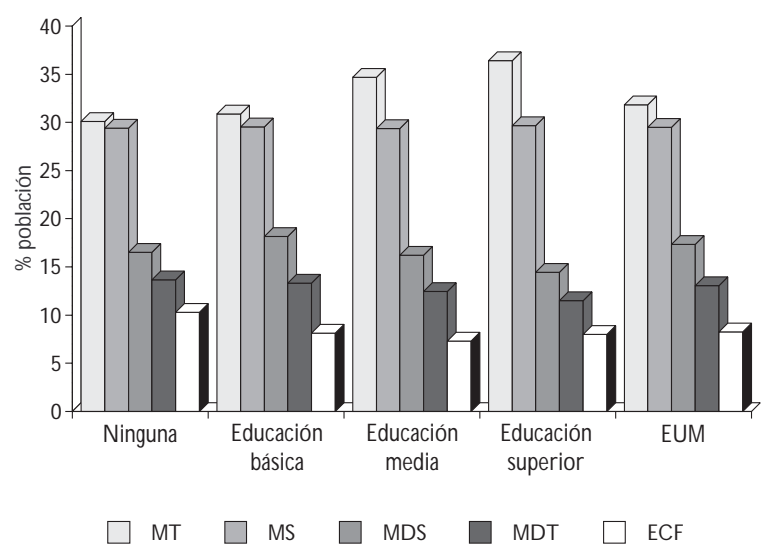

EUM: Estados Unidos Mexicanos

MT:mejorar el trato dentro del SS

MS: mejorar la salud de la población

MDS: minimizar diferencias en la población respecto a la salud

MDT: minimizar desigualdades en la capacidad de respuesta del SS

ECF: lograr la equidad en la contribución financiera del SS

Fuente: Encuesta $N$ acional de Evaluación del Desempeño, 2002. Dirección General de Información y Evaluación del D esempeño; Secretaría de Salud

Figura 2. IMPORTANCIA OTORGADA A LOS OBJETIVOS DEL SISTEMA DE SALUD POR NIVEL DE EDUCACIÓN. MÉXICO, 2002
2002, se constata al observar que $5.7 \%$ de la población encuestada en el área rural considera su estado actual de salud como malo y $30 \%$ como regular, cifras superiores a las del área urbana (5.3 y $28 \%$, respectivamente) $(P r=0.000)$. También se reconoció que en el área rural $3.5 \%$ de la población no recibió atención médica a pesar de requerirla, número mayor al porcentaje del área urbana, que fue de $2.1 \%$ ( $P r=0.000)$ (cuadro I). Lo anterior implica que la percepción del estado de salud actual y la falta de atención médica en estas zonas son factores que modifican la valoración de la importancia de los objetivos del SS.

La relevancia que asigna la población a los objetivos del SS de acuerdo con el sexo es igual a la valoración general nacional, si bien entre las mujeres el porcentaje que considera al objetivo de mejorar el trato como el más importante fue superior al de los hombres (32.4 y $31 \%$, respectivamente). Por el contrario, el porcentaje relacionado con el objetivo de mejorar la salud de la población fue $5.1 \%$ más alto entre los hombres y $10.5 \%$ más respecto del objetivo de lograr la equidad en la contribución financiera. En virtud de estas diferencias, y con base en la prueba de la ji cuadrada $(P r=0.000)$, se observó que la variable del sexo alteraba la determinación de la importancia de los objetivos analizados (cuadro I).

En lo que respecta a la valoración de los objetivos del SS a través de la variable de la edad, se identificó que no existe, en términos generales, variación con la posición obtenida en el plano nacional. Sin embargo, al analizar la importancia otorgada a cada objetivo 


\section{Cuadro 1}

\section{Variables relacionadas con la importancia otorgada a los objetivos del SS. México, 2002}

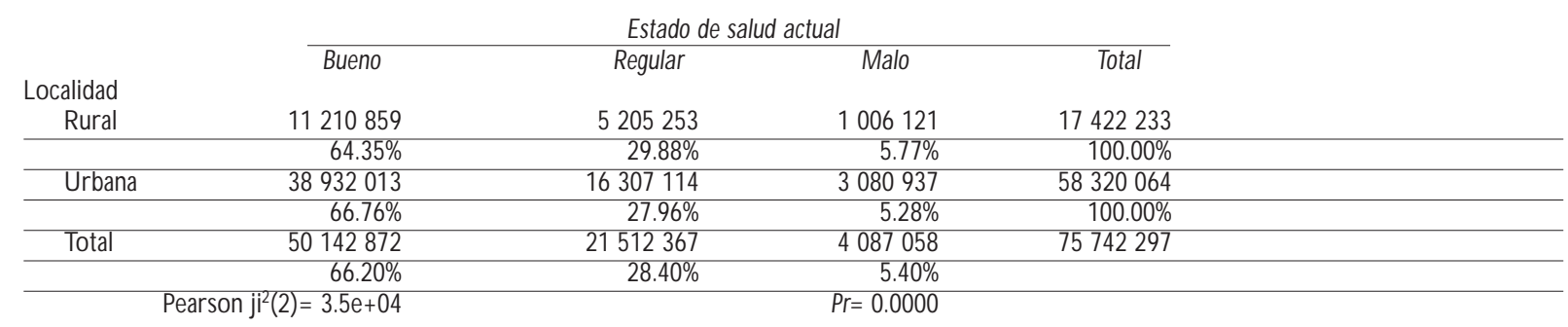

¿La última vez que necesitó atención médica la recibió?

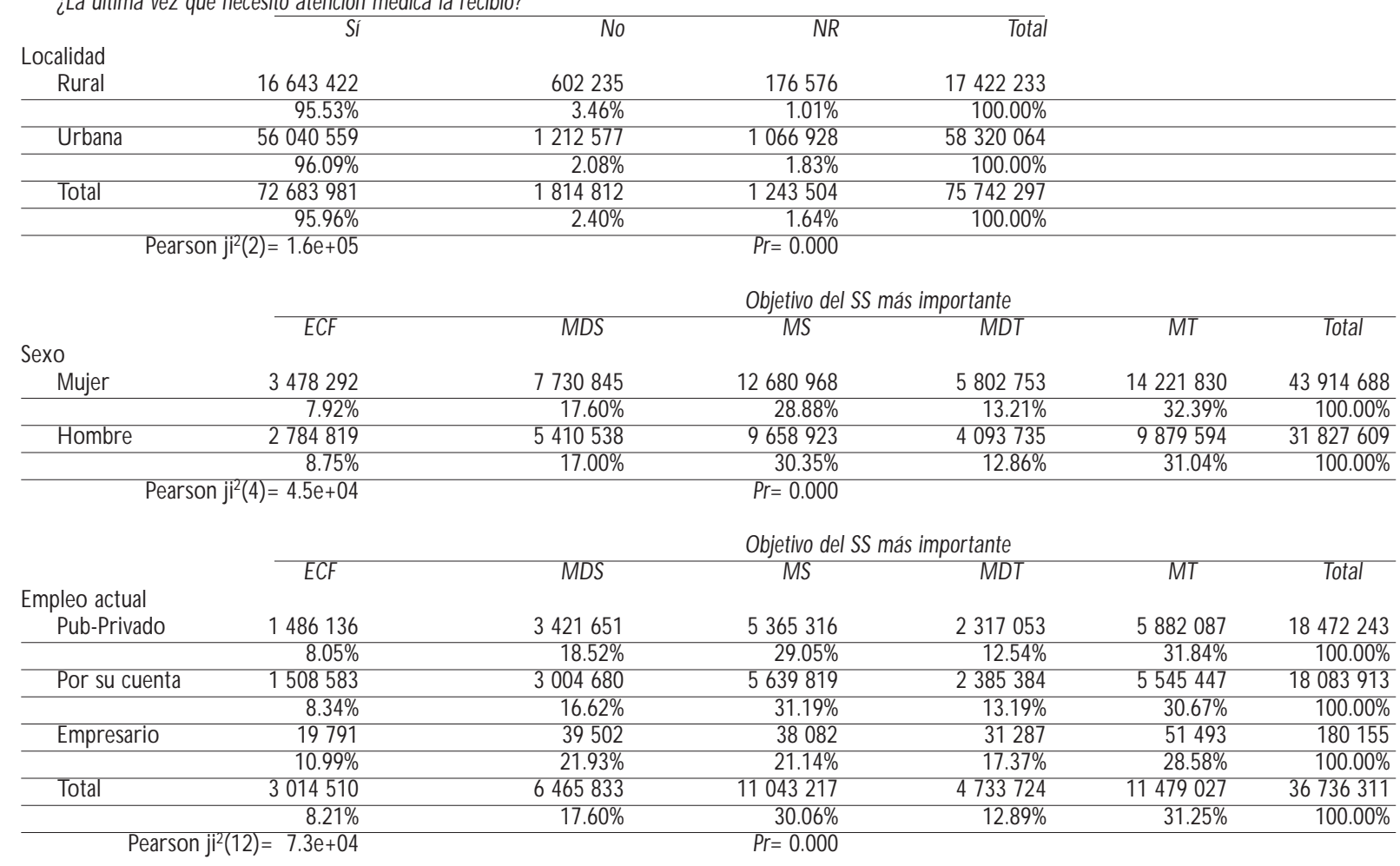

¿La última vez que necesitó atención médica la recibió?

\begin{tabular}{cccc} 
¿La ultima vez que necesito atención médica la recibió? & \\
\hline No & NR & Total
\end{tabular}

\begin{tabular}{|c|c|c|c|c|}
\hline \multirow[t]{2}{*}{ Satisfecho } & 10215666 & 124603 & 112985 & 10453254 \\
\hline & $97.73 \%$ & $1.19 \%$ & $1.08 \%$ & $100.00 \%$ \\
\hline \multirow[t]{2}{*}{ Regular } & 7944452 & 168729 & 111994 & 8225175 \\
\hline & $96.59 \%$ & $2.05 \%$ & $1.36 \%$ & $100.00 \%$ \\
\hline \multirow[t]{2}{*}{ Insatisfecho } & 5119207 & 181742 & 52441 & 5353390 \\
\hline & $95.63 \%$ & $3.39 \%$ & $0.98 \%$ & $100.00 \%$ \\
\hline \multirow[t]{2}{*}{ Total } & 23279325 & 475074 & 277420 & 24031819 \\
\hline & $96.87 \%$ & $1.98 \%$ & $1.15 \%$ & $100.00 \%$ \\
\hline
\end{tabular}

\footnotetext{
* Percepción de la asistencia médica en el país entre la población que considera que mejorar el trato es el objetivo más importante

Análisis a partir de la ji²

NR: No sabe o no responde

MS: Mejorar la salud de la población

MDS: Minimizar diferencias en la población respecto a la salud

MT: Mejorar el trato dentro del SS

MDT: Minimizar desigualdades en la capacidad de respuesta del SS

ECF: Lograr la equidad en la contribución financiera del SS
}

Fuente: Encuesta N acional de Evaluación del Desempeño 2002, Dirección General de Información y Evaluación del Desempeño, Secretaría de Salud 
según el grupo de edad en relación con la variable de grado de satisfacción con la salud, se advirtió que el grupo de mayores de 60 años considera más importante el objetivo de mejorar la salud, sea entre los que se declaran satisfechos con su salud o entre los que están insatisfechos (31 y $30.6 \%$, respectivamente); en consecuencia, se registraron diferencias de relevancia estadística entre los resultados analizados $(P r=0.000)$. Considerar más importante al objetivo de mejorar la salud entre este grupo es coherente si se toma en cuenta que, según la encuesta, 41.1 y $13.8 \%$ califican su salud actual como regular y mala, respectivamente, y porque $21.7 \%$ se halla insatisfecho con su salud. En ambos casos se obtuvieron diferencias estadísticas significativas $(P r=0.000)$.

El nivel educativo de la población de estudio también se analizó para determinar si influía en la valoración de los objetivos del SS. En términos generales, dentro de las categorías de esta variable, la posición concedida a los objetivos del SS no señaló cambios respecto de la posición nacional; empero, los porcentajes de población que consideran al objetivo de mejorar el trato dentro del SS como el más importante dentro de las categorías educación media $(34.7 \%)$ y educación superior $(36.4 \%)$, superaron al porcentaje de nivel nacional (31.8\%); esto implica que a mayor nivel educativo, mayor preferencia por mejorar la capacidad de respuesta del SS (figura 2). De esta forma, se reconoce que el nivel educativo de la población modifica la valoración de los objetivos del sistema; la prueba de la ji cuadrada deja ver que existen diferencias de relevancia estadística entre los grupos $(P r=0.000)$.

Otro hallazgo obtenido al analizar la posición asignada a los objetivos del SS, según el nivel educativo y el sexo, mostró que entre las mujeres, en todas las categorías de nivel educativo, el objetivo más importante del SS era mejorar el trato, mientras que para los hombres sin educación formal y educación básica el objetivo más importante fue mejorar la salud de la población ( $P r=$ 0.000 ), lo cual confirma que el trato dentro del SS se valora más cuanto mayor sea el nivel de escolaridad.

Por otra parte, al utilizar la variable de empleo actual de la población, los porcentajes asignados a los objetivos del SS entre los que trabajaban y los que no lo hacían al momento de la encuesta muestran el mismo orden de importancia que la cifra nacional. Sin embargo, al enfocar el análisis en la población que trabajaba al momento de la encuesta (36 millones $736 \mathrm{mil}$ 311 habitantes), se advirtió que entre los que trabajan por su cuenta (18 083 913), 31.2\% consideraba al objetivo de mejorar la salud como el más importante del SS, antes que mejorar el trato dentro del sistema (30.7\%). En las categorías empleado público-privado y empre- sarios, la posición de los objetivos fue la misma que la nacional (cuadro I). La prueba de la ji cuadrada registra diferencias estadísticas significativas entre las diversas categorías de esta variable $(P r=0.000)$; en consecuencia, con ello se rechaza la hipótesis de que la posición asignada a los objetivos del SS es la misma, sin importar cuál sea la variable que se analice.

Con la finalidad de identificar los factores que influyen en la población que trabaja de modo independiente para considerar más importante al objetivo de mejorar la salud, en relación con los otros objetivos analizados, se incluyeron en el análisis variables relacionadas con la salud y los servicios de atención. De esta forma, al analizar la percepción del estado de salud actual entre esta población, se observó que $69.9 \%$ de los que trabajan por su cuenta considera buena su salud, en tanto que los sujetos que consideraron su salud como regular y mala representaron $26.5 \%$ y $3.6 \%$, respectivamente. En contraste, entre la población integrada por empresarios que consideró al objetivo de mejorar la salud como el más importante del SS (38 082), 51.1\% cree que su salud actual es buena, $45.7 \%$ piensa que es regular y $3.3 \%$ que es mala. De esta forma, el efecto de la variable estado de salud actual, en combinación con el de la variable empleo actual, modifica la valoración de la población acerca de la importancia de los objetivos del SS, tras obtenerse diferencias estadísticas significativas para estas variables $(\mathrm{Pr}=0.000)$.

Al continuar con la identificación de los determinantes que pueden llevar a la población que trabaja de modo independiente a considerar más importante al objetivo de mejorar la salud, se analizó la necesidad de asistencia médica sin recibirla como variable. En este caso, 4.4\% requirió atención médica pero no la recibió; tal porcentaje es menor al informado entre los empleados públicos-privados (4.5\%) y mayor al de la población de empresarios (3.5\%). Cabe señalar que en estos dos últimos grupos se consideró más importante al objetivo de mejorar el trato dentro del sistema. La prueba de la ji cuadrada mostró que existen diferencias estadísticas significativas entre estas variables dentro de tales grupos; por lo tanto, para el grupo de trabajadores independientes no recibir atención médica al momento de necesitarla es un factor que altera su valoración de la importancia de los objetivos del SS, en especial para considerar al objetivo de mejorar la salud de la población como el más importante.

Por otra parte, la variable satisfacción con su salud se analizó a fin de establecer si influía en la valoración de la población respecto de la importancia de los objetivos del SS. Los resultados muestran que entre las categorías a) satisfecho, b) regular y c) insatisfecho con su salud, el orden de los objetivos fue el mismo que el 
expresado en general por la población nacional; no obstante, al analizar los porcentajes obtenidos, se reconoció que entre la población que dijo estar satisfecha de forma regular con su salud (16 millones 7 mil 258 personas), $18.8 \%$ señala como más importante al objetivo de mejorar el trato dentro del SS, en comparación con los que le dieron más relevancia al objetivo de mejorar la salud de la población (26.9\%). En las categorías de satisfecho e insatisfecho con su salud, las diferencias entre los porcentajes para uno y otro objetivos fueron de $4.8 \%$ y $7 \%$, respectivamente. Lo anterior muestra que para la población satisfecha de cierta forma con su salud es más relevante el objetivo de mejorar el trato dentro del SS, y a la vez confirma que la variable satisfacción con la salud influye entre los distintos grupos comparados al momento de valorar la importancia de los objetivos del SS, dado que la prueba de la ji cuadrada reveló diferencias estadísticas significativas entre ellos.

Con el fin de delinear aún más los factores que influyen en la población satisfecha de cierta forma con su salud para hacerles preferir el objetivo de mejorar la capacidad de respuesta dentro del sistema, se incluyó en el análisis la variable autopercepción del estado de salud actual. Se identificó entonces que $51.1 \%$ de los individuos cree que su salud es regular, $11 \%$ piensa que es mala y sólo $37.9 \%$ la considera buena (resultados coherentes si se toma en cuenta que se trata del grupo que muestra una regular satisfacción con su salud). En comparación, dentro del grupo satisfecho con su salud y entre los que declararon estar insatisfechos, los porcentajes para estado de salud actual regular fueron de 19.7 y $32.3 \%$, respectivamente, y para estado actual de salud malo de 1.9 y $11 \%$, también de modo respectivo. Las diferencias recogidas tuvieron relevancia estadística $(\mathrm{Pr}=0.000)$, lo que implica que la percepción del estado de salud actual, en combinación con la satisfacción de la salud, modifica la valoración de la población sobre los objetivos del SS.

Con posterioridad, se incorporó otra variable (última ocasión que requirió atención médica y no la recibió). Se reconoció así que el contacto de la población con los servicios, a pesar de notificar diferencias estadísticas significativas ( $P r=0.000)$, no es el más importante de sus determinantes al valorar la importancia de los objetivos del SS, puesto que el porcentaje de población que no recibió atención médica, a pesar de requerirla, es similar entre la población satisfecha, de cierta forma, con su salud y la insatisfecha, dentro del segmento que considera que mejorar el trato es el objetivo más relevante.

Por otra parte, de acuerdo con la ENED 2002, 11.5\% de la población que expresó insatisfacción con su salud consideró más importante al objetivo de conse- guir la equidad en la contribución financiera dentro del SS; tal porcentaje coloca a este objetivo en la cuarta posición y desplaza al objetivo de minimizar las desigualdades en la capacidad de respuesta del SS. Para identificar las determinantes que influían en esta población para que ocurriera este cambio, se analizó la variable autopercepción del estado de salud actual; de ese modo se identificó que $6.8 \%$ consideraba su estado de salud actual como malo y $23.7 \%$ como regular, resultados ambos inconsistentes con el hecho de que se trata de una población que refirió insatisfacción con su salud. Esto permite considerar que la percepción de esta población para preferir un sistema de salud que garantice la equidad en la contribución financiera no depende de su estado de salud actual. Por esta razón, y con el fin de explicar qué otras variables pudieran alterar su valoración, se analizó la estructura de población por grupo de edad. Los resultados mostraron que en los grupos de 17 a 24 años y 25 a 59 años se mantuvo en cuarto lugar la equidad en la contribución financiera, mientras que en el grupo de 60 años y más, el cuarto sitio lo ocupó el objetivo de minimizar las desigualdades en la capacidad de respuesta del sistema. Por lo tanto, entre la población en edad productiva es más importante contar con un sistema que se financie de manera equitativa, siempre que la aportación de la población se realice con base en su capacidad económica.

Para sustentar este argumento se analizó la variable empleo actual, cuyas categorías fueron las siguientes: trabaja y no trabaja al momento de realizar la ENED 2002. Con los resultados se observó que, a pesar de que este segmento forma parte de la población económicamente activa (es decir, todas las personas de 12 años o más que en la semana de referencia realizaron algún tipo de actividad económica o formaban parte de la población desocupada abierta), $45.3 \%$ del grupo de 17 a 24 años y $47.1 \%$ del de 25 a 59 años -que estaban insatisfechos con su salud y que sitúan en cuarto lugar al objetivo de lograr la equidad en la contribución financiera- no trabajaban al momento de la encuesta $(P r=0.000)$. Lo anterior explica, por una parte, por qué se considera importante dicho objetivo en estos grupos de población y, por otra, permite pensar que no contar con un empleo remunerado es un factor que limita las posibilidad para acceder a servicios de atención médica u obliga a realizar gastos onerosos para atender su salud.

En vista de lo anterior, además del análisis de las variables satisfacción con la salud, edad y trabajo actual, también se incorporó otra variable: requirieron atención médica y no la recibieron. Así se advirtió que $3.1 \%$ del grupo de 17 a 24 años (9 656 personas) y $5.2 \%$ 
del grupo de 25 a 59 años (53 586) no obtuvo servicios a pesar de necesitarlos. Con esto se establece que la variable empleo actual, y su relación con la variable requirieron atención médica y no la recibieron, es un elemento que influye en los grupos de población insatisfechos con su salud durante su valoración de la importancia de los objetivos del SS. Las pruebas de la ji cuadrada efectuadas durante estos análisis permitieron observar la existencia de diferencias estadísticas significativas $(P r=0.000)$.

Por último, la valoración de los objetivos del SS a partir de la variable satisfacción con el funcionamiento de la atención médica en el país mostró que la posición asignada a los objetivos es igual que la notificada en la población nacional. Al comparar los porcentajes de población para cada objetivo del SS dentro de cada una de las categorías de esta variable, se identificó que $27.8 \%$ más de la población que expresó insatisfacción con la atención médica consideró más importante al objetivo de mejorar el trato en comparación con los que eligieron al objetivo de mejorar la salud de la población. En el grupo que refirió cierta satisfacción con la asistencia médica, la diferencia entre estos dos objetivos fue de $7.38 \%$, en tanto que la diferencia en la población satisfecha con la asistencia fue de $0.45 \%$ en favor del objetivo de mejorar el trato dentro del SS.

Para reconocer los posibles determinantes que influyen en la población insatisfecha con la asistencia médica del país, y que considera más importante al objetivo de mejorar el trato dentro del SS (5 millones 353 mil 390 personas), se analizó otra variable más (última ocasión que necesitó atención médica y no la recibió). Entonces se advirtió que 3.4\% no recibió atención médica a pesar de requerirla. En comparación, entre la población que eligió mejorar el trato dentro del sistema y mostró satisfacción con la asistencia médica (10 millones 453 mil 254), sólo $1.1 \%$ no recibió servicios, mientras que de la población medianamente satisfecha con la asistencia, 2.1\% no recibió atención médica (cuadro I). Con base en la prueba de la ji cuadrada se determinó que la variable satisfacción con la asistencia médica del país modifica la valoración de la importancia de los objetivos del SS entre las categorías estudiadas, y que la falta de atención, a pesar de necesitarla, es uno de los determinantes de esta selección.

Como se observa, el empleo de la prueba ji cuadrada durante el análisis suministró elementos para rechazar la hipótesis según la cual la posición asignada por la población a los objetivos del SS es la misma, al margen de las variables empleadas en la comparación, dado que se obtuvieron diferencias estadísticas significativas $(P r=0.000)$. Más aún, su uso permitió identificar las variables que se incorporaron al modelo de regresión, cuyo objetivo, por un lado, fue establecer la correlación de las variables independientes incluidas en el análisis en relación con la importancia que la población concede a los objetivos del SS y, por el otro, constatar que dichas variables pueden considerarse determinantes cuando la población valora la importancia de los objetivos del SS analizados en este estudio.

\section{Modelo de regresión logística multinomial}

Se aplicó un modelo de regresión logística multinomial (politómica) con objeto de establecer los efectos de las variables independientes sobre la variable dependiente obtenida a través de la pregunta "¿cuál objetivo del SS le parece el más importante?", la cual tiene cinco respuestas categóricas que no implican un orden establecido. En el modelo se consideró como categoría de referencia al objetivo de mejorar la salud de la población, puesto que se lo considera el definitorio del SS y ocupa la primer posición dentro del PRONASA 2001-2006. El modelo explica en 49\% la variabilidad en la valoración de los objetivos del SS. Cabe señalar que la estimación de los coeficientes se interpreta como una reducción del riesgo relativo $(R R R)$.

En el cuadro II se presentan los resultados completos obtenidos en el modelo, si bien la descripción, por motivos de espacio, se enfocó en correlacionar el efecto de las variables independientes con los objetivos de mejorar el trato dentro del SS (objetivo más importante según la ENED 2002) y mejorar la salud de la población (segundo en importancia en la ENED 2002 y categoría de comparación), toda vez que, como se observó, las variaciones se presentaron casi siempre entre estos dos objetivos del SS. A partir de ello se observó que la probabilidad de que la población urbana prefiera el objetivo de mejorar el trato dentro del SS en comparación con el de mejorar la salud es $24 \%$ más alta que la probabilidad de las personas del área rural, resultado que refuerza el hallazgo de que la población rural prefería más al objetivo de mejorar la salud, como se mencionó con anterioridad. Por otra parte, en relación con el nivel educativo, la probabilidad de preferir el objetivo de mejorar el trato en lugar de mejorar la salud entre la población con educación media y superior es 10 y $16 \%$ más alta, respectivamente, respecto de la probabilidad para la población sin educación; tal correlación proporciona sentido a las diferencias identificadas entre el porcentaje de población que valora al objetivo de mejorar el trato dentro del sistema como el más importante en relación con el objetivo de mejorar la salud, como se muestra en la figura 2.

A partir de la condición laboral de la población, según el modelo, la probabilidad de que la que traba- 


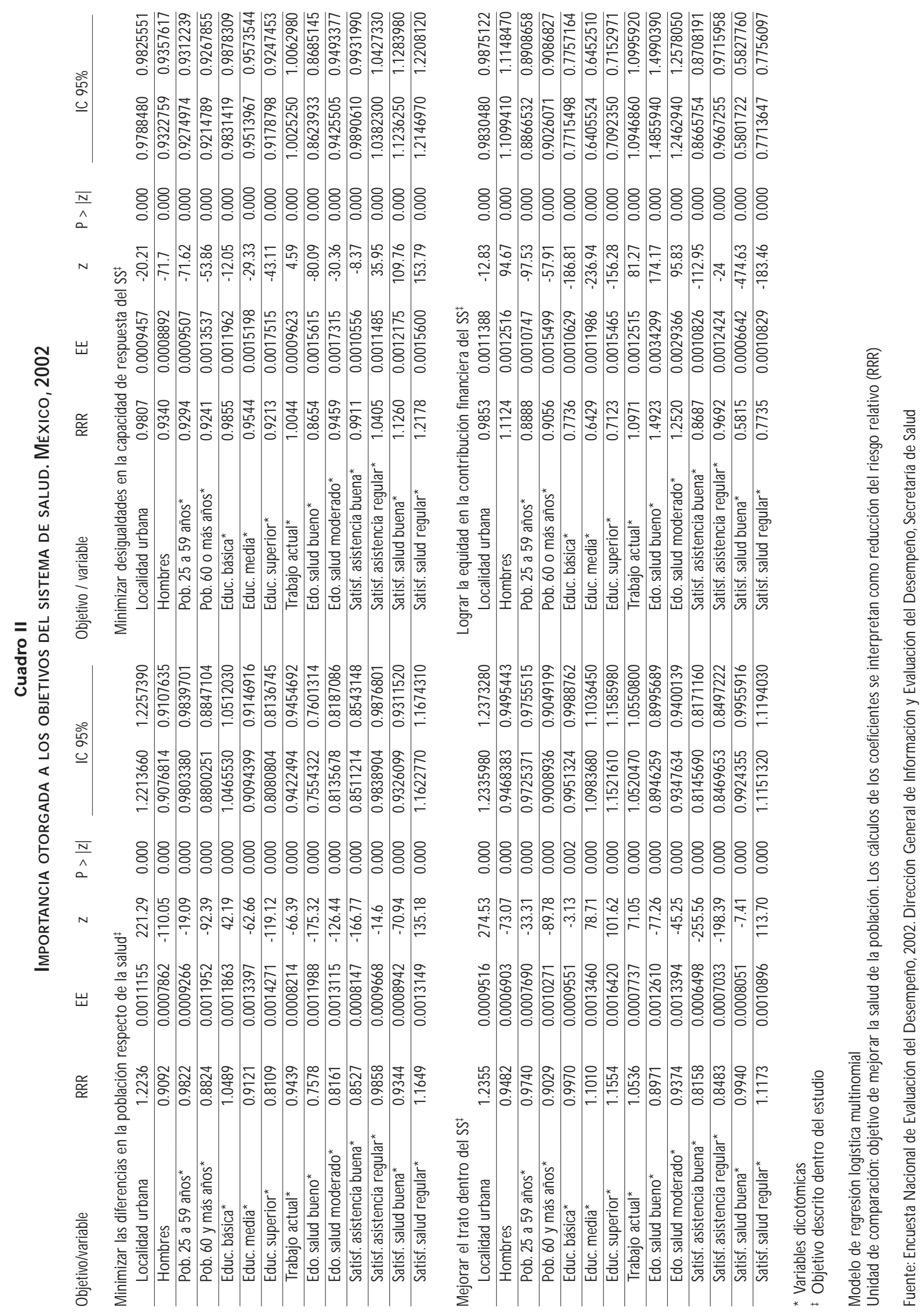


jaba prefiriera el objetivo de mejorar el trato dentro del SS, en vez del de mejorar la salud, es $5 \%$ más elevada que la probabilidad de aquellos que no trabajaban al momento de la encuesta, un resultado que sustenta los hallazgos sobre la influencia que tiene el aspecto laboral en la valoración de la importancia de los objetivos del SS. Por otra parte, entre la población que refirió estar satisfecha en cierta forma con su salud, la probabilidad de preferir el objetivo de mejorar el trato y no el de mejorar la salud es $12 \%$ más alta que la probabilidad de quienes están insatisfechos con su salud.

Por otra parte, la probabilidad de que los hombres consideren al objetivo de mejorar el trato dentro del sistema como el más importante, en oposición al de mejorar las condiciones de salud, es $5 \%$ menor respecto de la probabilidad entre las mujeres. De hecho, como se observó, la diferencia entre el porcentaje de hombres y mujeres que consideran al objetivo de mejorar las condiciones de salud como el más importante es de $5.1 \%$ en favor de los hombres.

La edad, como se comentó, es una variable que influye al momento de valorar la importancia que tienen para la población los objetivos del SS, en particular en el grupo de 60 años y más, ya que de acuerdo con el modelo, la probabilidad de que este grupo de población considere al objetivo de mejorar el trato como el más importante, en vez del de mejorar la salud, es $10 \%$ menor que la probabilidad que posee la población de 17 a 24 años y $7 \%$ menor que la probabilidad de la población de 25 a 59 años. Por otro lado, también se reconoció que la probabilidad de que la población satisfecha con la atención médica en el país considere a la mejoría en el trato como el objetivo más importante, en lugar del objetivo de mejorar la salud, es 19\% menor que la probabilidad del grupo que se considera insatisfecho con la asistencia; es decir, a pesar de que en ambos grupos el trato adecuado es el principal objetivo, su influencia entre los que están insatisfechos es aún mayor que entre los satisfechos. Lo mismo ocurre con el grupo que señaló estar satisfecho de mediana forma con la atención médica, dado que la probabilidad de que consideren que mejorar el trato es el principal objetivo, en lugar del de mejorar las condiciones de salud, es $16 \%$ más baja que la probabilidad de los que están insatisfechos. Por último, se advierte que en las variables restantes incluidas en el modelo no existe una probabilidad más alta que otra, puesto que se ubican dentro del valor nulo o de igualdad que es de uno.

\section{Discusión}

En el PRONASA 2001-2006 se establece que no puede haber progreso general sin un sistema de salud que responda a las aspiraciones de los mexicanos; también se señala que la democratización de la salud, entre otras cosas, significa estimular la participación de los ciudadanos en todos los niveles del sistema, desde asumir la responsabilidad por su propia salud, hasta influir en el diseño de la agenda sectorial y la toma de decisiones. Por lo tanto, valorar la percepción de la población sobre los objetivos que persigue el SS e identificar los determinantes que modifican tal percepción son una tarea esencial para conocer sus expectativas y proporcionar elementos que favorezcan la intervención de la sociedad en la conducción del sistema, lo cual apoya la democratización del sector salud.

De esta forma, el objetivo de este estudio fue documentar de forma empírica la importancia que tienen para la población los cinco objetivos intrínsecos del actual SS en México y describir algunos de los determinantes que participan en su valoración, con base en los datos de la ENED 2002; el objetivo es aportar elementos para definir prioridades y cumplir dichos objetivos.

A partir de los resultados se observó que, en términos generales, la población analizada en este estudio consideró más importante al objetivo de mejorar el trato dentro del sistema de salud, lo cual difiere del planteamiento establecido en el PRONASA 2001-2006, en el cual el objetivo más importante es mejorar la salud de los mexicanos, seguido por el objetivo de abatir las desigualdades en salud. Lo anterior permite establecer que para un volumen considerable de población, contar con un sistema de salud que respete la dignidad de las personas y ofrezca servicios orientados al usuario es una aspiración impostergable. Sin embargo, cabe señalar que durante este análisis se advirtió que la importancia concedida a los objetivos puede variar según sean el grupo analizado, sus características y la influencia que tienen diversos factores externos. También se observó que esta variación se presentó sobre todo entre el objetivo de mejorar el trato dentro del SS y el de mejorar la salud de la población. Con base en ello, destacan los siguientes hallazgos:

- La percepción de la condición de salud actual, junto con otras características de la población, es una variable que influye en grado notorio cuando se valora la importancia de los objetivos del SS. Lo anterior se constató en 12 entidades federativas, en las áreas rurales y entre la población mayor de 60 años, en las que se detectó que el estado de salud actual se percibía como malo o regular; en consecuencia, se identificó una mayor preferencia por el objetivo de mejorar la salud de la población. 
- Además, en las áreas rurales la falta de atención médica, pese a necesitarla, fue un factor que actuó para que esta población considerara más importante al objetivo de mejorar la salud. Tal hallazgo se apoya en los resultados del modelo matemático que señala que en las áreas urbanas la probabilidad de preferir al objetivo de mejorar el trato dentro del SS fue $24 \%$ más alta en comparación con la probabilidad en las áreas rurales.

- Al analizar la población de acuerdo con el sexo se identificó que para hombres y mujeres el objetivo más importante era el de mejorar el trato dentro del SS; no obstante, al analizar los porcentajes de población que consideraban al objetivo de mejorar la salud como el más importante, se observó que entre los hombres había una mayor preferencia por este objetivo en comparación con las mujeres (5.1\% más). De igual manera, al combinar el efecto de la variable sexo con el nivel educativo se reconoció que entre las mujeres, sin importar cuál fuera el nivel de escolaridad, se mantenía la preferencia por mejorar el trato, mientras que entre los hombres sin educación formal y con educación básica el objetivo más importante fue el de mejorar la salud.

- En relación con lo anterior, los resultados muestran que la preferencia por el objetivo de mejorar el trato dentro del SS es mayor cuanto más alto sea el nivel educativo .

- Otro hallazgo relevante fue que entre la población que trabajaba de forma independiente durante la aplicación de la encuesta parecía más importante el objetivo de mejorar la salud, lo cual, como se describió, se relacionaba con la percepción del estado de salud actual y la falta de atención al momento de requerirla.

- Por último, la población insatisfecha con la atención médica del país muestra una mayor preferencia por el objetivo de mejorar el trato. Este dato es consistente si se considera que $3.4 \%$ de esta población no recibió atención médica a pesar de necesitarla, una cifra mayor al porcentaje de la población que señaló estar satisfecha con la asistencia en el país y que no recibió atención médica $(1.1 \%)$.

A partir de estos resultados, y como parte del objetivo planteado en este estudio (aportar elementos para la definición de prioridades y cumplir estos objetivos), resulta necesario considerar que la valoración que hace la población sobre la importancia de los objetivos del SS, y en general de su operación, se vincula con la experiencia de ésta durante su interacción con lo servicios de salud y la modifican características personales y ambientales, lo que hace difícil establecer argumentos concluyentes. Sin embargo, una forma de homogeneizar algunos de sus argumentos consiste en establecer y fortalecer los canales de comunicación y participación que permitan a la población conocer cuál es el rumbo del SS, la forma en que opera y el modo de atender las necesidades y expectativas. Esto permitiría, por una parte, que la población conociera y exigiera sus derechos y, por la otra, que asumiera su compromiso con la salud y participara de manera informada en la conducción del SS.

El fortalecimiento de este proceso de comunicación debe estar acompañado de acciones concretas en materia de atención, trato adecuado y protección financiera, al considerar que existen diferencias entre los grupos poblacionales -documentadas en este estudioque obligan a que las acciones y las expectativas de la población sean congruentes. Por ejemplo, en las áreas rurales es necesario realizar mayores esfuerzos para abatir los rezagos en salud, en tanto que la atención a las personas mayores de 60 años debe fortalecerse como respuesta a la percepción de que lo más importante para el sistema debe ser mejorar la salud de la población.

Por otra parte, la población con un mayor nivel educativo otorga más importancia al objetivo de mejorar el trato en comparación con la población de menor nivel educativo (que considera que mejorar la salud debe ser el objetivo principal del SS). Esto destaca la existencia de desigualdades que se reflejan en las expectativas, las cuales debería evitar el SS, al proporcionar las mismas oportunidades de atención y el mismo trato, al margen de la ubicación geográfica de la población, su situación económica, nivel de educación o cualquier otra característica. De esta forma, los resultados de este estudio permiten conocer las expectativas de la población sobre el sistema de salud al que aspiran, y tener elementos para identificar dónde es necesario fortalecer la operación del SS y en qué áreas.

Asimismo, es preciso comentar algunas de las limitantes enfrentadas durante la realización de esta investigación:

1. En la encuesta no se incluyó alguna pregunta que suministrara información sobre el conocimiento previo que tenía la población seleccionada acerca de los objetivos del sistema de salud en México. La valoración que efectuó la población pudo estar sustentada sólo en la explicación que recibieron de los encuestadores.

2. No se documentó la existencia de estudios similares en los que se analizara la importancia de los 
objetivos del SS y permitieran tener puntos de comparación, pese a que los ejercicios apoyados por la OMS en diversos países incluyeron este tema.

La realización de este estudio debe considerarse un primer acercamiento a este tema, dado que es necesario profundizar, aplicar otras metodología de análisis y comparar los resultados con alguna experiencia internacional, con la finalidad de proporcionar información relevante que apoye la operación de los sistemas de salud y su crecimiento.

\section{Referencias}

1.W alt G. Health policy: an introduction to process and power. Londres: Zed Books, 1994:240.

2. Presidencia de la República. Plan N acional de Desarrollo 2001-2006. México: Presidencia de la República, 2001:46-47.

3. Frenk J. La salud de la población. Hacia una nueva salud pública. La ciencia desde México/133. México: Fondo de Cultura Económica, 1994:98-108
4. 0 rganización Mundial de la Salud. Informe sobre la salud en el mundo 2000. Mejorar el desempeño de los sistemas de salud. Punto 3 del orden del día provisional. A53/4.53a A samblea Mundial de la Salud. Ginebra: O MS, 2000.

5. Frenk J, Lozano R, González-Block MA et al. Economía y salud: propuestas para el avance del sistema de salud en México. Informe final. México: Fundación Mexicana para la Salud, 1994:64-69.

6. Murray CJL, Frenk J.A framework for assessing the performance of health systems. Bull W orld Health O rgan 2000;78(6):717-731.

7. 0 rganización Panamericana de la Salud. Evaluación y mejora del desempeño de los sistemas de salud en la región de las Américas. W ashington: 0 rganización Panamericana de la Salud. 0 ficina Sanitaria Panamericana. 0 ficina Regional de la 0 rganización Mundial de la Salud, 2001.

8. Secretaría de Salud. Programa N acional de Salud 2001-2006. La democratización de la salud en México. Hacia un sistema universal de salud. 3a. edición. México: Secretaría de Salud, 2001:73-82.

9. Secretaría de Salud. Reglamento interior de la Secretaría de Salud. México: Diario 0 ficial de la Federación, 2004:49.

10. Secretaría de Salud. Salud: México 2002. Información para la rendición de cuentas. México: Secretaría de Salud. Dirección General de Información y Evaluación del Desempeño, 2003:56-61.

11. Palma-C oca 0 , 0 laiz-Fernández G. Metodología de la Encuesta $\mathrm{N}$ acional de Evaluación del Desempeño. Salud Publica Mex 2005;47(Supl 1):S66-S74. 